\title{
Echocardiographic characteristics of pulmonary arterial hypertension in children with horizontally transmitted HIV
}

\author{
Anca Meda Georgescu ${ }^{1, A-F}$, Cosmin Moldovan ${ }^{2, C-F}$, Janos Szederjesi, ${ }^{3, C-F}$, Dan Georgescu ${ }^{4, B-F}$, Leonard Azamfirei ${ }^{3, A, ~ C-F}$ \\ ${ }^{1}$ Infectious Diseases Clinic, University of Medicine and Pharmacy of Tirgu Mures, Romania \\ 2 Department of Histology, University of Medicine and Pharmacy of Tirgu Mures, Romania \\ ${ }^{3}$ Intensive Care Clinic, University of Medicine and Pharmacy of Tirgu Mures, Romania \\ ${ }^{4} 7^{\text {st }}$ Internal Medicine Clinic, University of Medicine and Pharmacy of Tirgu Mures, Romania \\ A - research concept and design; B - collection and/or assembly of data; C - data analysis and interpretation; \\ $D$ - writing the article; $E$ - critical revision of the article; $F$ - final approval of article
}

\section{Address for correspondence}

Cosmin Moldova

E-mail:cosmin.moldovan@outlook.com

Funding sources

None declared

Conflict of interest

None declared

Received on 0ctober 20, 2015

Revised on December 13, 2015

Accepted on March 17, 2016

DOI

$10.17219 /$ acem $/ 62244$

\section{Copyright}

Copyright by Author(s)

This is an article distributed under the terms of the

Creative Commons Attribution Non-Commercial License

(http://creativecommons.org/licenses/by-nc-nd/4.0/)

\begin{abstract}
Background. The prevalence of primitive pulmonary arterial hypertension (PAH) in patients with human immunodeficiency virus infection (HIV) is estimated at approximately $0.5 \%$, significantly higher than in the general population.

Objectives. This study aimed to assess the echocardiographic modifications in HIV-associated pulmonary arterial hypertension (PAH).

Material and methods. A group of 117 patients, aged under 16, with horizontally transmitted HIV staged according to the U.S. Center for Disease Control and Prevention criteria, were included in this prospective study, with echocardiographic abnormalities in 79 children. The study group consisted of 27 HIV-infected patients with PAH, while the control group consisted of 38 patients with normal ultrasound features. The diagnostic criterion for PAH was the presence of a mean pulmonary artery pressure above $25 \mathrm{~mm} \mathrm{Hg}$, determined at 2 consecutive measurements having at least 6 months distance between them. All subjects underwent a complex echocardiographic assessment, including assessment of left and right ventricular hypertrophy and evaluation of left ventricular function, associated with determination of the immunological stage.
\end{abstract}

Results. We recorded the presence of PAH in 27 patients (23.08\%), in whom an average value of $31.48 \mathrm{~mm} \mathrm{Hg}$ was recorded for pulmonary artery pressure. All patients had mild forms of PAH. Age, gender and immunological stage showed no significant differences in the PAH group compared to patients in the control group. Right ventricular hypertrophy was encountered in $95.23 \%$ and left ventricular hypertrophy in 88.88\% of the patients with PAH. Left ventricular dysfunction, a complication of pulmonary hypertension, was relatively rare (11.11\%).

Conclusions. In children with HIV infection, PAH is present in a relatively mild form and does not correlate with the clinical and immunological stage of HIV infection, evolving as a seemingly primitive condition.

Key words: children, pulmonary arterial hypertension, HIV, ultrasonography 


\section{Introduction}

The prevalence of primitive pulmonary arterial hypertension (PAH) in patients with human immunodeficiency virus infection (HIV) is estimated at approximately $0.5 \%$, significantly higher than in the general population. The improvement in the survival rates of these patients, as a result of the introduction of highly active antiretroviral treatment (HAART) and of a better control of infectious intercurrences, increases the associated transmission risks and the importance of this pathology. ${ }^{1}$ Similarly to primitive pulmonary arterial hypertension, the condition associated with HIV infection has a very complex pathogenesis, being characterized by abnormal vasomotor response of the pulmonary vascular bed, pulmonary angiogenesis disorders and disorders of cellular ion channels. ${ }^{2}$ Restrictive and obstructive ventilatory dysfunction caused by recurrent pulmonary infections, hypoxia and secondary pulmonary hypertension, other viral infections, and cocaine and intravenous drug use can be added. ${ }^{3}$

According to European guidelines, PAH is defined as an increase in mean pulmonary arterial pressure above $25 \mathrm{~mm} \mathrm{Hg}$ at rest, as assessed by cardiac catheterization. The present guidelines of the European Society of Cardiology indicate the invasive determination of pulmonary artery pressure using right heart catheterization as the gold standard for establishing the diagnosis of $\mathrm{PAH}^{4}$ However, we should not forget that cardiac catheterization is associated with a significantly higher risk of HIVinfection transmission in this population, therefore the routine use of this method, especially for those patients, should be avoided. Instead, echocardiography (a non-invasive and repeatable method) presents numerous advantages (safe, non-invasive, painless, inexpensive), becoming an extremely useful tool not only for screening but also for monitoring the disease, while other non-invasive tests such as clinical examination, X-ray or ECG can only raise the suspicion of $\mathrm{PAH}$.

The objective of this study was to assess the echocardiographic characteristics of PAH in children with horizontally transmitted HIV.

\section{Patients and methods}

Our study is a prospective, longitudinal and cohort study, conducted over a period of 5 years in the Infectious Diseases Clinic I, Tirgu Mures, Romania.

The study population was comprised of children with diagnosed HIV infection monitored by the aforementioned center. The inclusion criteria were the presence of HIV infection with documented horizontal transmission and age under 16 years. The exclusion criteria were the presence of congenital cardiac malformations, vertical transmission of the HIV infection and lack of cooperation with ultrasound examination during the study. The patient population consisted of $117 \mathrm{HIV}$-infected children in whom cardiac ultrasound was performed at baseline and repeated during the 5-year follow-up (at 6-month intervals), divided into 2 groups: 79 children with echocardiographic abnormalities and 38 children without echocardiographic abnormalities. The study group consisted of $27 \mathrm{HIV}$-infected patients with PAH, while the control group consisted of 38 patients with normal ultrasound features.

The PAH study group was defined taking into account the dominant PAH pathology. Cases where PAH was secondary to other cardiac pathology were excluded.

The diagnosis of the HIV infection was confirmed based upon 2 second generation ELISA tests for HIV1 and HIV2 antibodies and confirmed by Western Blot.

Clinical and immunological staging, needed for stratification of the study population and for assessment of the correlations between the severity of cardiac involvement and the stage of the HIV infection, was done using the 1993 CDC Centers for Disease Control and Prevention (CDC) Atlanta criteria. ${ }^{5}$

The echocardiographic examination was performed using M mode (mono-dimensional), 2D (2-dimensional), spectral Doppler and color Doppler echocardiography. The following parameters characterizing the ventricular function and structure were monitored: left and right ventricular mass, left ventricular ejection fraction and fractional shortening, presence and severity of valvular regurgitation at aortic, mitral, pulmonary and tricuspid levels, ventricular wall thickness (tricuspid annular plane systolic excursion - TAPSE). Pulmonary artery pressure measurements in the study group were performed using Bernoulli's equation applied to the regurgitant flow across the tricuspid valve in systole. In the case of associated pulmonary regurgitation, color Doppler measurement of the regurgitant flow across the pulmonary valve made possible the assessment of diastolic pressure in the pulmonary artery. The ultrasound study of the pulmonary valve sometimes made possible diagnosis of $\mathrm{PAH}$.

The diagnostic criterion for PAH was the presence of a mean pulmonary artery pressure above $25 \mathrm{~mm} \mathrm{Hg}$ at rest, determined at 2 consecutive measurements having at least 6 months distance between them. ${ }^{4}$

We considered the measurements performed in the absence of acute or chronic respiratory infections when the restrictive or obstructive dysfunction of the pulmonary parenchyma may have led to hypoxia and changes in pulmonary vascular resistance. No adjustments were required for body weight criteria in children. ${ }^{6}$

\section{Statistical analysis}

Statistical analysis was performed with GraphPad InStat 2003 software. The data was considered statistically significant for $\mathrm{p}<0.05$. Mean, minimum, and maximum 
values, standard deviation and confidence intervals were calculated for all the parametric data samples. Gaussian distribution was determined using the KolmogorovSmirnov formula, the value obtained having to be higher than 0.10. If the obtained value was lower, we changed the parameter data into nonparametric data or we used tests for nonparametric data (Mann-Whitney test) for the analysis of two independent samples.

For the statistical analysis of parametric values we used Student's t test to analyze 2 dependent or independent samples, and we used the t test with Welch's correction when significant differences were recorded in the standard deviations of the study.

Nonparametric data samples were statistically analyzed using Fisher's exact test for smaller groups and $\chi^{2}$ test for larger groups, with or without Yates's correction, $\mathrm{p}<0.05$ being considered significant. Correlation between non-parametric unpaired samples was calculated using the Spearman correlation coefficient. In the case of paired data, the Wilcoxon test was used.

The study was approved by the institution's Ethics Committee.

\section{Results}

\section{Epidemiological data}

The gender distribution in the entire study group was balanced (49.58\% males $/ 50.42 \%$ females). The mean age of the children with HIV infection in the study group was $14.03 \pm 0.23$ years.

Analysis of the mean age of the children in the 2 groups did not show statistically significant differences between the groups $(15.03 \pm 0.28$ years in the group with pulmonary hypertension vs $15.21 \pm 0.41$ years in the control group).

In the PAH group we found a higher prevalence of the disease in female patients (17 patients, 62.96\%) than in male (10 patients, $37.03 \%$ ). However, the gender distribu- tion of patients did not differ significantly from the control group (24 patients, $63.16 \%$ and 14 patients, $36.84 \%$ ) (Fisher's test $\mathrm{p}=1.00$ ).

\section{Staging of HIV infection}

According to CDC Atlanta criteria the distribution into groups of clinical and immunological severity staging (LT-CD4 depletion) did not show statistically significant differences between the groups ( $p=0.51$ for clinical staging and $\mathrm{p}=0.296$ for immunological staging). ${ }^{5}$

\section{Echocardiographic changes in the study groups}

The examinations in the study group revealed multiple echocardiographic aspects. The recorded changes were cumulative for the duration of the study and were not always evident on the first evaluation of the patient. We recorded echocardiographic abnormalities in a number of 79 children $(67.52 \%)$ from the study group. As previously mentioned, the changes observed were left ventricular systolic dysfunction, left ventricular hypertrophy, pulmonary hypertension, right ventricular hypertrophy, dilated cardiomyopathy and pericardial collections. Thirty-eight $(32.48 \%)$ patients had normal ultrasound features over the entire period and were designated as the control group.

Variable PAH were encountered in 16 other patients out of the 79 with echocardiographic abnormalities; all these patients had dilated cardiomyopathy. The diagnostic criteria in this lot and, respectively, the criteria for exclusion from the PAH study group were: ejection fraction below $40 \%$ and increased left ventricle end-systolic and enddiastolic diameters. ${ }^{6}$ Although the increase of the mean pressure in the pulmonary artery was significant in this lot (mean value $34.31 \mathrm{~mm} \mathrm{Hg}$ ), patients with dilated cardiomyopathy were not included in the PAH study group, as in their case the pressure change was considered to be a consequence of the systolic dysfunction pertaining to dilated cardiomyopathy (Fig. 1).

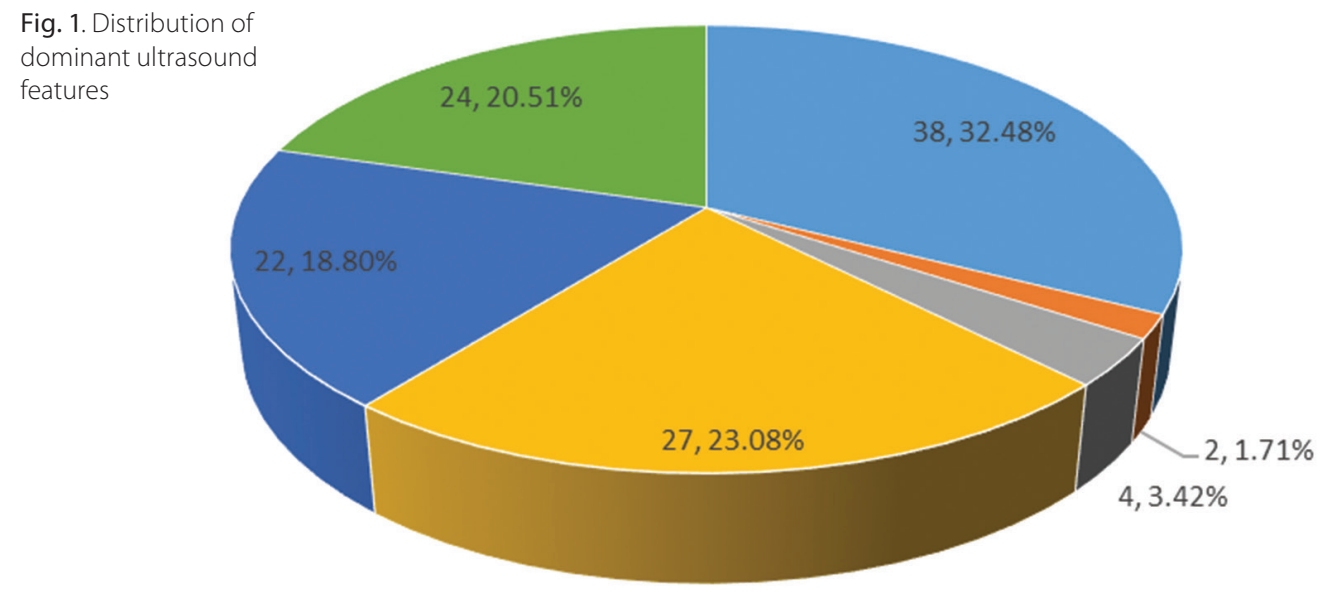

- Normal features

- Exsudative pericarditis

- Dilatative cardiomyopathy 
Table 1. Clinical and immunological staging of patients with PAH vs patients in the control group

\begin{tabular}{|c|c|c|c|c|c|c|}
\hline \multirow{2}{*}{$\begin{array}{l}\text { Clinical and } \\
\text { immunological } \\
\text { categories }\end{array}$} & \multicolumn{3}{|c|}{ Clinical staging } & \multicolumn{3}{|c|}{ Immunological staging } \\
\hline & A & B & C & 1 & 2 & 3 \\
\hline PAH group & 14 & 12 & 1 & 14 & 11 & 2 \\
\hline Control group & 16 & 18 & 4 & 21 & 10 & 7 \\
\hline Fisher's exact test & \multicolumn{3}{|c|}{$p=0.51$} & \multicolumn{3}{|c|}{$p=0.296$} \\
\hline
\end{tabular}

The echocardiographic characteristics of patients in the group diagnosed with PAH and those in the control group are summarized in Table 2.

Mean pulmonary arterial pressure was $31.48 \pm 1.74 \mathrm{~mm} \mathrm{Hg}$, compared to $17.33 \pm 2.24 \mathrm{~mm} \mathrm{Hg}$ in the control group $(\mathrm{p}<0.0001)$.

Analyzing the severity of the

\section{Features of the PAH group}

Twenty-seven patients, representing $23.08 \%$ of the entire pediatric group studied, constituted the subgroup of patients with $\mathrm{PAH}$.

The evaluation of the clinical and immunological status of HIV infection in patients with PAH compared to the control group, did not show statistically significant differences regarding the clinical or immunological staging (Table 1). pulmonary arterial hypertension syndrome in relation to the mean values of pulmonary arterial pressure $(25-40 \mathrm{~mm} \mathrm{Hg}=$ mild, $41-70 \mathrm{~mm} \mathrm{Hg}=$ moderate, over $70 \mathrm{~mm} \mathrm{Hg}=$ severe), we observed that all children with pulmonary hypertension associated with HIV infection showed mild forms. No more than 15 patients had values above $30 \mathrm{~mm} \mathrm{Hg}$, of which 8 had repeated mean values above $37 \mathrm{~mm} \mathrm{Hg}$.

Due to increased resistance in the pulmonary vascular bed, lower flow velocities were recorded in the pul-

Table 2. Echocardiographic characteristics in children and adolescents with horizontally acquired HIV infection and PAH vs. the control group

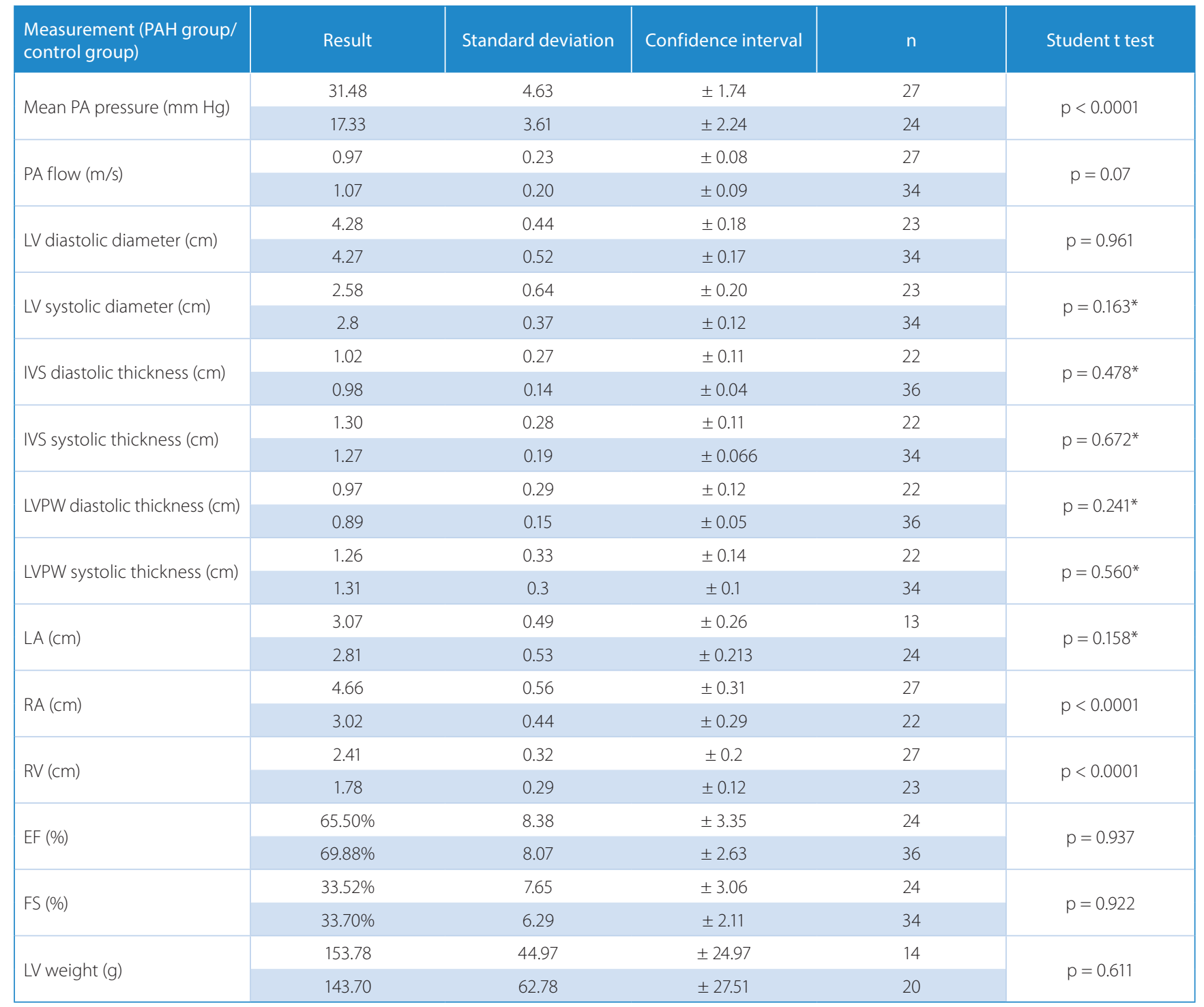

PA - pulmonary artery; LV - left ventricle; IVS - interventricular septum; PW - posterior wall; LA - left atrial diameter; RA - right atrial diameter; RV - right ventricle diameter; EF - ejection fraction; FS - fractional shortening; *Welch correction. 
monary artery trunk, the mean value in the study group being $0.97 \pm 0.08 \mathrm{~m} / \mathrm{s}$ vs $1.07 \pm 0.09 \mathrm{~m} / \mathrm{s}$ in the control group. However, the difference was not statistically significant because the pursued hemodynamic parameter is not important for a positive diagnosis or for the severity of the syndrome.

We did not note statistically significant differences between left ventricular diastolic diameter in children with pulmonary hypertension compared to the control group ( $p=0.9610)$. Similarly, left ventricular systolic diameter did not differ significantly between the 2 groups ( $p=0.163$ ). The analysis of the left ventricular wall thickness in patients with HIV infection associated with pulmonary hypertension showed normal values. Thus, the mean diastolic interventricular septum thickness did not differ significantly between the 2 groups $(\mathrm{p}=0.478)$ and neither did the mean systolic interventricular septum thickness differ significantly between the 2 groups $(\mathrm{p}=0.672)$.

The posterior wall diastolic thickness of the left ventricle did not differ significantly between the 2 groups $(p=0.241)$ and neither did the systolic posterior wall thickness present statistically significant differences $(\mathrm{p}=0.560)$.

The recorded diameter of the left atrium in the 2 groups indicated normal values and no statistically significant differences ( $\mathrm{p}=0.158$ ).

Measurements indicated a greater size of the right atrium than normal, relative to body weight, in 16 of the patients in the study group, representing $59.25 \%$. The mean diameter of the right atrium had higher values in patients with pulmonary hypertension than in those in the control group, and the difference was highly significant statistically ( $\mathrm{p}<0.0001)$.

Ultrasound examination revealed right ventricular dilatation in 25 of the children in the study group, representing $92.59 \%$.

The mean diameter of the right ventricle was much greater in the group of children with PAH compared to those in the control group, and the difference was highly significant statistically $(\mathrm{p}<0.001)$.

As a result of the absence of any morphological and functional alteration of the left ventricle in patients with pulmonary hypertension, there were no modifications observed at the level of the aortic valve (Table 3 ).

Thus, the aortic and mitral insufficiency did not differ significantly in frequency in the 2 groups studied $(14.8 \%$ vs $10.52 \%, \mathrm{p}=0.709$, and $7.40 \%$ vs $0 \%, \mathrm{p}=0.168$, respectively). In all cases, color Doppler echocardiography revealed minor aortic insufficiency, with minimal regurgitation.

Increased pressure in the pulmonary vascular bed did not lead to a higher prevalence of pulmonary valvular insufficiency $(40.74 \%$ vs $42.10 \%, \mathrm{p}=1.00)$. While pulmonary insufficiencies were mild in the control group, con- sidered normal variants, we recorded 4 cases of moderate pulmonary insufficiency and 2 cases of severe pulmonary insufficiency (Table 3) in patients with PAH.

At least moderate tricuspid regurgitation was detected in the majority of patients with pulmonary hypertension associated with HIV infection (25 patients, 92.59\%). The presence of a significant tricuspid regurgitation is an important sign of pulmonary hypertension and makes possible the measurement of the mean pressure in the pulmonary artery with the aid of the Doppler examination if there is any pulmonary valve stenosis. Differences between the groups in the severity of tricuspid regurgitation are highly significant statistically $(\mathrm{p}<0.0001)$. (Table 3$)$ 
help of these new technologies, such as tricuspid annular plane systolic excursion (TAPSE), tricuspid annular systolic velocity $\left(\mathrm{S}^{\prime}\right)$, right ventricular myocardial performance index (MPI) and right ventricular fractional area changes (FAC), have been demonstrated to be correlated with the severity of the PAH and the underlying heart disease, as indicators of the global function of the right ventricle, and are nowadays largely used for evaluation of children with $\mathrm{PAH}^{8,9}$ However, they might have a lower value for the assessment of the regional function in different segments of the ventricles. In such cases, information provided by the speckle tracking technology might help in identifying the pattern of ventricular wall motion, based on a determination of free wall longitudinal peak systolic strain and right ventricular global longitudinal peak systolic strain as markers of right ventricular deformation associated with progression of the PAH. ${ }^{10,11}$

However, similar to our study, recently published literature data indicates that conventional echo parameters characterizing the right ventricle function, as determined with $2 \mathrm{D}$ echocardiography, proved to remain valuable to assess the severity of PAH in children with congenital heart diseases. ${ }^{12}$ Since in our study the etiology of the PAH was related to the presence of HIV infection, we did not record major congenital heart diseases in these patients, therefore the assessment of the regional function of the right ventricle could be considered less important than the assessment of the global function. Therefore, the parameters determined by $2 \mathrm{D}$ echocardiography and routine Doppler assessment, like in our study, could be considered valuable enough to represent a standard routine test for screening of the presence of PAH in the HIVinfected children population.

Other studies report lower values of the prevalence of the disease: severe forms in $4.9 \%$ of cases, in a group of children with peripartum exposure to HIV infection. ${ }^{13}$ The prevalence of primitive PAH associated with HIV was estimated at $0.5 \%$ in the pre-HAART stage ${ }^{14}$, a level that remains unchanged at $0.46 \%$, and post-HAART ${ }^{15}$, the data provided being highly suggestive of the ineffectiveness of this therapy regarding the incidence and severity of PAH associated with HIV infection. ${ }^{4}$ The most pertinent explanation regarding the high differences in the prevalence cited above, is the difference related to the diagnostic methods used. ${ }^{15}$

On the other hand, it is possible that the results of the present study on PAH in children and adolescents with HIV infection record differences relative to those reported in similar studies, due to the unique epidemiological characteristic of the studied group, which consisted of patients belonging to a pediatric group with horizontally acquired HIV infection at a young age, in the late 1980s, due to a well-known epidemiological accident in Romania. The infection of this pediatric group with the F1 subtype of HIV is also special, the subtype representing less than $1 \%$ of the strains circulating globally. ${ }^{16}$
In the present study, LT-CD4 depletion was not a risk factor for the development of PAH syndrome. The literature also indicates the lack of a statistically significant correlation between pulmonary hypertension associated with HIV infection and the stage of HIV cell immunosuppression, although some authors report the value of LT-CD4 as an independent factor in the prognosis of survival in PAH associated with HIV infection and a more severe evolution of the syndrome in patients in the AIDS stage of the disease. ${ }^{16}$

Ultrasound examination revealed right ventricular dilatation in $92.59 \%$ of patients in the PAH group, in whom the mean diameter of the right ventricle was significantly higher compared to those in the control group. This feature is part of the ultrasound picture characteristic of this condition, which does not present particularities in HIV infected children and adolescents. It is worth mentioning that in the study group there was a subgroup of patients with dilated cardiomyopathy or isolated right ventricular dilatation without elevated pressure in the pulmonary artery. On the other hand, out of 79 patients with echocardiographic abnormalities, there were patients with systolic dysfunction, dilated cardiomyopathy, and other dominant pathologies. Those patients can also manifest a degree of ultrasound-identifiable pulmonary hypertension. ${ }^{17}$

A careful reporting to body weight is required to establish normal or pathological left ventricular dimensions. ${ }^{6}$ Pathological values were recorded in 2 patients who had mild concentric hypertrophy of the posterior and septum wall. The data presented indicates a lack of association between pulmonary hypertension syndrome and left ventricular hypertrophy. This finding is important because left ventricular hypertrophy is one of the most important cardiac changes in HIV infection with well-studied pathogenetic mechanisms. ${ }^{18}$ The lack of this association most likely indicates the intervention of different pathogenetic mechanisms or an individual susceptibility to developing pulmonary hypertension. ${ }^{19}$ Some data in the literature indicates susceptibility determined by certain genes of the major complex of histocompatibility in causing pulmonary hypertension syndrome ${ }^{20}$ in which pathogenesis is intricate and multifactorial, and with the most frequently-mentioned pathogenetic mechanisms responsible for being vasculitic changes caused by the mediators of the inflammation and by the interaction of viral proteins with the molecular structure of the host which stimulates the production of endothelin-1 and platelet growth factor. ${ }^{1,2}$

We did not find alterations in the myocardial contractile function in the PAH group, despite the fact that the presumed pathogenetic mechanisms of both this syndrome and those of myocardial dysfunction have many common links, represented mostly by the immune response to the released inflammatory mediators in response to HIV infection. ${ }^{15,21}$ On the other hand, there are 
studies in adults that report a prevalence of $25 \%$ of left ventricular dysfunction in patients with pulmonary hypertension associated with HIV infection. ${ }^{22}$

An important limitation of this study is the lack of comparison between echocardiographic data and catheterization data, knowing that there is a variation between echocardiographically and hemodynamically assessed pulmonary artery pressure and that the diagnosis of PAH is established with the use of right heart catheterization according to current guidelines. ${ }^{4}$

\section{Conclusions}

Pulmonary hypertension syndrome in children with horizontally acquired HIV infection is common and it evolves as a seemingly primitive condition of mild severity without being influenced by the clinical and immunological stage of the HIV infection.

\section{References}

1. Sitbon O. HIV-related pulmonary arterial hypertension: Clinical presentation and management. AIDS. 2008;22(Suppl 3):S55-62.

2. Klings ES, Farber HW. The pathogenesis of HIV-associated pulmonary hypertension. In: Barbaro G, Karger, ed. HIV Infection and the Cardiovascular System. Basel. 2003;71-82.

3. Pellicelli AM, Palmieri F, Cicalini S, Petrosillo N. Pathogenesis of HIVrelated pulmonary hypertension. Ann NY Acad Sci. 2001;946:82-94.

4. Galie N, Hoeper MH, Humbert M, et al. Guidelines for the diagnosis and treatment of pulmonary hypertension. Eur Heart J. 2009;30:2493-2537.

5. Kamps BS, Hoffmann C. CDC Classification System. In: Hoffmann C Rockstroch JK, Kamps BS, eds. HIV Medicine. Paris: Flying Publisher. 2005:27-28.

6. Feigenbaum H. Echocardiography. Lippincott Williams \& Wilkins, 1994;658-681.

7. Cicalini $S$, Chinello $P$, Cicini MP, Petrosillo N. Pulmonary arterial hypertension and HIV infection. AIDS. 2008;22:2219-2220.

8. Koestenberger M, Ravekes W, Everett A, et al. Right ventricular function in infants, children and adolescents: Reference values of the tricuspid annular plane systolic excursion (TAPSE) in 640 healthy patients and calculation of z-score values. J Am Soc Echocardiogr. 2009;22:715-719.
9. Koestenberger M, Nagel B, Ravekes W, et al. Reference values of the tricuspid annular peak systolic velocity in healthy pediatric patients, calculation of z-score values, and comparison to the tricuspid annular plane systolic excursion. Am J Cardiol. 2011;109:116-121.

10. Van der Hulst AE, Roest AA, Delgado V, et al. Relationship between temporal sequence of right ventricular deformation and right ventricular performance in patients with corrected tetralogy of Fallot. Heart. 2011;97:231-236.

11. Levy PT, Mejia AAS, Machefsky A, et al. Normal ranges of right ventricular systolic and diastolic strain measures in children: A systematic review and meta-analysis. JASE. 2014;27:549-560.

12. Kassem E, Humpl T, Friedberg MK. Prognostic significance of 2-dimensional, M-mode and Doppler echo indices of right ventricular function in children with pulmonary arterial hypertension. Am Heart J. 2013;165:1024-1031.

13. Diógenes MS, Succi RC, Machado DM, Moisés VA, Novo NF, Carvalho AC. Cardiac longitudinal study of children perinatally exposed to human immunodeficiency virus type 1. Arg Bras Cardiol. 2005;85:233-240.

14. Speich R, Jenni R, Opravil M, Pfab M, Russi EW. Primary pulmonary hypertension in HIV infection. Chest. 1991;100:1268-1271.

15. Sitbon O, Lascoux-Combe C, Delfraissy JF, et al. Prevalence of HIVrelated pulmonary arterial hypertension in the current antiretroviral therapy era. Am J Respir Crit Care Med. 2008;177:108-113.

16. Hemelaar J, Gouws E, Ghys PD, Osmanov S. Global and regional distribution of HIV-1 genetic subtypes and recombinants in 2004. AIDS. 2006;20:13-23.

17. Georgescu AM, Moldovan C, Azamfirei L, Georgescu D. Ultrasound and histopathological features of myocardial involvement in HIV infection in children. Rom J Morphol Embryol. 2014;55:3-6.

18. Degano B, Guillaume M, Savale L, et al. HIV-associated pulmonary arterial hypertension: Survival and prognostic factors in the modern therapeutic era. AIDS. 2010;24:67-75.

19. Zuber JP, Calmy A, Evison JM, et al. The Swiss HIV cohort study group pulmonary arterial hypertension related to HIV infection: Improved hemodynamics and survival associated with antiretroviral therapy. Clin Infect Dis. 2004;38:1178-1185.

20. Morse JH, Barst RJ, Itescu S, et al. Primary pulmonary hypertension in HIV infection: An outcome determined by particular HLA class II alleles. Am J Respir Crit Care Med. 1996;153:1299-1301.

21. Papita AM, Albu A, Fodor D, Bondor C, Itu C, Carstina D. Markers of preclinical vacular disease and left ventricular diastolic dysfunction in patients with HIV infection. Med Ultrason. 2012;14:10-18.

22. Isiguzo GC, Okeahialam BN, Danbauchi SS, Odili AN, Iroezindu MO, Placi U. Contributions of pulmonary hypertension to HIV-related cardiac dysfunction. Indian Heart J. 2013;65:644-649. 